\title{
Spatial mapping of fluorophore quantum yield in diffusive media
}

Yanyu Zhao

Darren Roblyer

\section{SPIE.}




\title{
Spatial mapping of fluorophore quantum yield in diffusive media
}

\author{
Yanyu Zhao and Darren Roblyer* \\ Boston University, Department of Biomedical Engineering, 44 Cummington Mall, Boston, Massachusetts 02215, United States
}

\begin{abstract}
Fluorescence quantum yield (QY) indicates the efficiency of the fluorescence process. The QY of many fluorophores is sensitive to local tissue environments, highlighting the possibility of using QY as an indicator of important parameters such as $\mathrm{pH}$ or temperature. QY is commonly measured by comparison to a well-known standard in nonscattering media. We propose a new imaging method, called quantum yield imaging (QYI), to spatially map the QY of a fluorophore within an optically diffusive media. QYI utilizes the wide-field diffuse optical technique spatial frequency domain imaging (SFDI) as well as planar fluorescence imaging. SFDI is used to measure the optical properties of the background media and the absorption contributed by the fluorophore. The unknown $Q Y$ is then calculated by combining information from both modalities. A fluorescent sample with known QY is used to account for instrument response. To demonstrate QYI, rhodamine B and SNARF-5 were imaged in liquid phantoms with different background optical properties. The methanol:water ratio and $\mathrm{pH}$ were changed for rhodamine B and SNARF-5 solvents, respectively, altering the QY of each through a wide range. QY was determined with an agreement of 0.021 and 0.012 for rhodamine B and SNARF-5, respectively. (C) 2015 Society of Photo-Optical Instrumentation Engineers (SPIE) [DOI: 10.1117/1.JBO.20.8.086013]
\end{abstract}

Keywords: quantum yield; fluorescence imaging; molecular imaging; diffuse optics; scattering media; photon migration.

Paper 150305R received May 7, 2015; accepted for publication Jul. 24, 2015; published online Aug. 26, 2015.

\section{Introduction}

Optical imaging with the use of exogenous targeted fluorescent agents (i.e., molecular imaging) is increasingly used in the preclinical setting for understanding the disease progression and the biological effects of treatments in vivo with high specificity. ${ }^{1}$ Probes that excite and emit in the red and near-infrared (NIR) $(600$ to $1000 \mathrm{~nm})$ allow for deep photon penetration (several mms) while minimizing tissue autofluorescence. ${ }^{2}$ Almost all commercially available fluorescence small animal imaging systems collect planer fluorescence light emission from the tissue surface. One of the major drawbacks of these imaging setups is the inability to extract quantitative fluorescence signals or probe concentrations due to the effects of the surrounding tissue optical properties, namely absorption and scattering. Without additional correction for background optical properties, these systems are unable to provide fluorescence intensity maps that are quantitatively comparable between samples or animals. An additional complicating factor is that the intensity of the collected fluorescence is not only dependent on the fluorophore concentration, but also depends on the quantum yield (QY), which is defined as the ratio of emitted photons to absorbed photons in the respective emission and absorption wavelengths bands. ${ }^{3}$ For some fluorophores, the QY is highly sensitive to environmental conditions such as $\mathrm{pH}$, temperature, oxygen concentration, and other factors. ${ }^{4-8}$ While a varying QY generally represents a challenge for quantitative fluorescence imaging, it also suggests that QY may be used as a novel microenvironmental biosensor if it can be accurately measured in vivo.

In the laboratory setting, fluorescence QY is commonly determined by comparing the fluorescence intensity of a fluorophore with an unknown QY to a standard with known QY, with both samples measured in an optically dilute media. ${ }^{9-12} \mathrm{QY}$ determination is more difficult in tissue due to the interaction of both the excitation and emission photons with the surrounding medium, where there may be substantial attenuation due to both absorption and scattering events. However, if the optical properties at both the absorption and emission wavelengths are known, established models allow for a quantitative fluorescence signal to be extracted. ${ }^{13-15}$ If one also assumes a known QY, quantitative fluorescence also allows for probe concentrations to be determined.

In this work, we present a new quantitative widefield imaging modality called quantum yield imaging (QYI) to first measure the probe concentration and then to determine an unknown probe QY. QYI uses a combination of spatial frequency domain imaging (SFDI) and planar fluorescence imaging to spatially map the QY of a fluorophore in diffusive media such as tissue. ${ }^{16}$ SFDI quantifies optical properties of the diffusive media at both the excitation and emission wavelengths, and is used to determine probe concentration using absorption contrast at the excitation wavelength. This information then feeds a modified quantitative fluorescence model that corrects the planar fluorescence raw signal intensity, producing a pixel-by-pixel map of QY values in a widefield image. ${ }^{14,15}$ We demonstrate this method using tissue-mimicking optical phantoms and show that the QY of two fluorophores, rhodamine B, which is highly sensitive to its solvent composition, and SNARF-5, which is $\mathrm{pH}$ sensitive, can be quantitated and spatially mapped using QYI under a variety of experimental conditions and background optical properties. QYI may provide a new means to nondestructively 
determine important tissue microenvironmental parameters in vivo, such as local $\mathrm{pH}$, temperature, and probe binding.

\section{Methods}

QYI combines SFDI and planar fluorescence with a modified empirical model of quantitative fluorescence. The details of each of these methods are outlined here as well as the overall methodology for combining their information outputs to generate QY maps. Then the methodology for determining the agreement of QYI is outlined, namely by extracting the QY of two fluorophores, rhodamine B and SNARF-5, each measured under a variety of experimental conditions.

SFDI is used to extract optical properties from the background media in QYI. SFDI utilizes spatially modulated sinusoidal light patterns of visible or NIR light, projected at different spatial frequencies and wavelengths, to separate the relative contributions of absorption and scattering in diffusive media. ${ }^{13,16}$ The light patterns are modulated by a digital micromirror device (DMD) spatial light modulator. Modulation patterns are projected onto the sample during the camera integration time, typically milliseconds to 100 s of milliseconds, dependent on wavelength and reflectance of the sample. The captured images are demodulated and calibrated to create diffuse reflectance maps. From these maps, optical properties (absorption and reduced scattering) can be extracted from each pixel in the image using a Monte-Carlo-based look-up table method. ${ }^{17}$ This methodology has been implemented in multiple in vivo studies and has potential applications in subsurface tomography. ${ }^{18-20}$ For this study, we used the OxImager RS SFDI system (Modulated Imaging Inc., Irvine, California). This system provides LED illumination up to 11 wavelengths spanning the visible to NIR and images with a $15 \mathrm{~cm} \times 15 \mathrm{~cm}$ field of view. A set of five

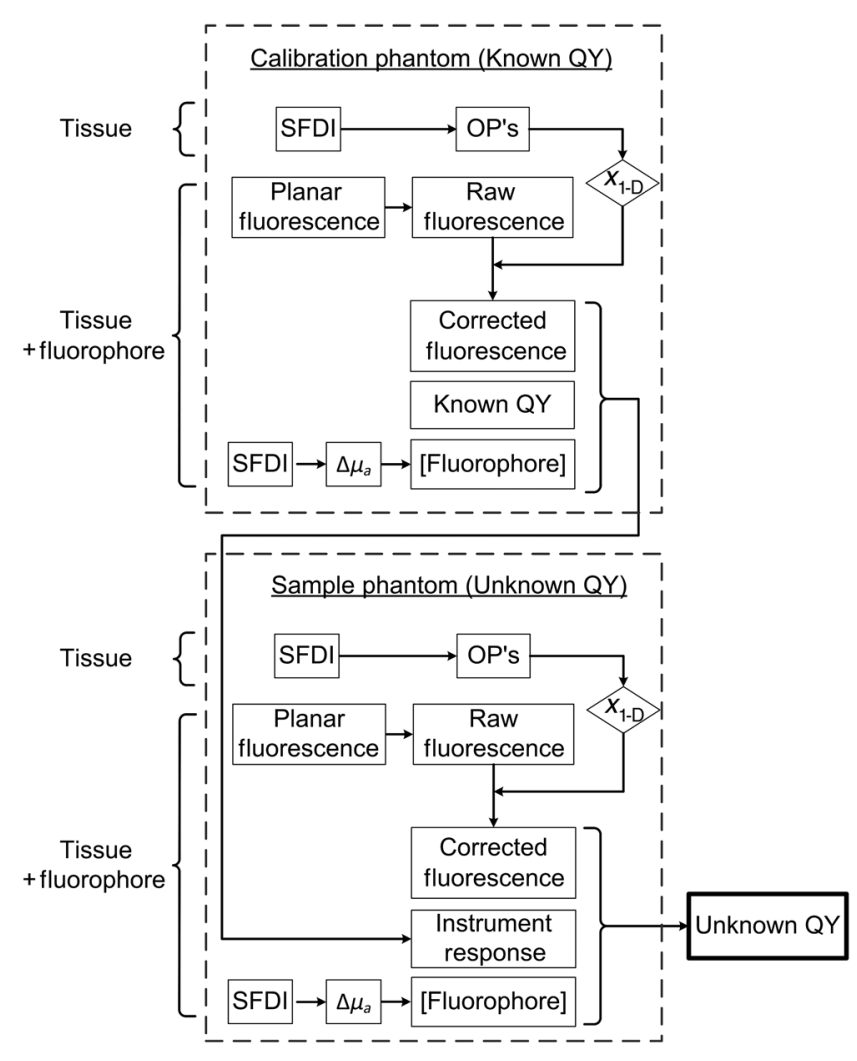

Fig. 1 Flowchart of quantum yield imaging data processing. spatial frequencies are utilized for the extraction of optical properties: $0,0.05,0.1,0.15$, and $0.2 \mathrm{~mm}^{-1}$. The imaging lens used in the system has a depth of field larger than $5 \mathrm{~cm}$. The system response is determined with the use of a calibration silicone phantom of known optical properties. For data processing, both height and angle correction of SFDI are applied. ${ }^{21}$ For this study, SFDI measurements were taken at $526 \mathrm{~nm}$, near the peak absorbance of both fluorophores tested, and at $591 \mathrm{~nm}$, in the emission band of the fluorophores. The same lightsource and camera were used to collect planar fluorescence images with the addition of a cleanup bandpass filter with a center wavelength at $534 \mathrm{~nm}$ and a 30-nm passband. A longpass filter with a cutoff wavelength of $561 \mathrm{~nm}$ was used in the emission band.

While there are a variety of models available to extract quantitative fluorescence, in this work, we utilized the model developed by Gardner et al. ${ }^{13-15}$ This model is an empirical approach based on semi-infinite, homogeneous Monte-Carlo simulations. It accounts for the presence of tissue absorption and reduced scattering, assumes planar illumination, and is simplified to a one-dimensional (1-D) problem. This model can be applied to an imaging geometry by separately analyzing each image pixel. ${ }^{18}$ SFDI provides $\mu_{a}$ and $\mu_{s}^{\prime}$ at both excitation and emission wavelengths, which are used as inputs to the model to calculate a fluorescence attenuation correction factor. This factor accounts for optical absorption and scattering, and is applied to a raw fluorescence planar image. The fluorescence correction factor $X_{1-\mathrm{D}}$ is determined as follows:

$$
\begin{aligned}
X_{1-\mathrm{D}}\left(\lambda_{\mathrm{ex}}, \lambda_{\mathrm{em}}\right)= & \frac{C_{1}\left(\lambda_{\mathrm{ex}}\right) C_{3}\left(\lambda_{\mathrm{em}}\right)}{k_{1}\left(\lambda_{\mathrm{ex}}\right) / \delta\left(\lambda_{\mathrm{ex}}\right)+k_{3}\left(\lambda_{\mathrm{em}}\right) / \delta\left(\lambda_{\mathrm{em}}\right)} \\
& -\frac{C_{2}\left(\lambda_{\mathrm{ex}}\right) C_{3}\left(\lambda_{\mathrm{em}}\right)}{k_{2}\left(\lambda_{\mathrm{ex}}\right) / \delta\left(\lambda_{\mathrm{ex}}\right)+k_{3}\left(\lambda_{\mathrm{em}}\right) / \delta\left(\lambda_{\mathrm{em}}\right)} .
\end{aligned}
$$

$C_{n}$ and $k_{n}$ are defined in Table 1 of Gardner et al. ${ }^{14}$ as empirical coefficients. They are dependent on the diffuse reflectance $R_{d}$. Since $R_{d}$ is a function of $\mu_{a}$ and $\mu_{s}^{\prime}$, it can be calculated from the values determined by SFDI measurement. $\delta$ refers to the optical penetration depth, which is calculated from $\mu_{a}$ and $\mu_{s}^{\prime}: \delta(\lambda)=$ $1 / \sqrt{3 \mu_{a}(\lambda)\left[\mu_{a}(\lambda)+\mu_{s}^{\prime}(\lambda)\right]}$.

A flowchart of QYI data processing is shown in Fig. 1. In general, the determination of an unknown QY requires knowledge of the fluorophore concentration, a planar fluorescence map corrected for background optical properties, and the instrument response, which is a function of detector sensitivity, imaging geometry, and other instrument related parameters. In order to determine the unknown QY of a sample, the instrument response is first determined from a calibration phantom with known QY and known fluorophore concentration (top box of Fig. 1). This instrument response is then applied to the sample, which contains the same fluorophore but with unknown QY (bottom box of Fig. 1). More specifically, first a calibration phantom is fabricated (with no exogenous fluorophore) and SFDI is used to quantify the background optical properties of the diffuse sample over a widefield area at both the absorption and emission wavelengths for a specific fluorophore. Then SFDI is used again to determine the additional absorption $\left(\Delta \mu_{a}\right)$ at the excitation wavelength caused by the addition of an exogenous fluorophore to the sample. This $\Delta \mu_{a}$ is used to determine the concentration of the added fluorophore based on the known extinction coefficient at the excitation wavelength. The solvent of the calibration phantom must be carefully controlled so that the fluorophore QY is known (e.g., the $\mathrm{pH}$ must be kept 
Table 1 Background optical properties of liquid phantoms (rhodamine B).

\begin{tabular}{ccc} 
& \multicolumn{2}{c}{ Excitation wavelength: $526 \mathrm{~nm}$} \\
\cline { 2 - 3 } Liquid phantom & $\mu_{a}\left(\mathrm{~mm}^{-1}\right)$ & $\mu_{s}^{\prime}\left(\mathrm{mm}^{-1}\right)$ \\
\hline Phantom set 1 & 0.028 & 0.55 \\
Phantom set 2 & 0.022 & 1.04 \\
Phantom set 3 & 0.051 & 0.53 \\
Phantom set 4 & 0.045 & 0.99 \\
Phantom set 5 & 0.074 & 0.51 \\
Phantom set 6 & 0.070 & 0.96 \\
\hline
\end{tabular}

constant). The same information is also extracted from a sample phantom (with unknown QY). The background $\mu_{a}$ and $\mu_{s}^{\prime}$ from both phantoms are then input into the Gardner model to produce fluorescence correction factors $X_{1-D}$. The fluorescence correction factor, calculated probe concentration, known QY, and the known fluorophore extinction coefficient at the excitation wavelength of the calibration phantom can be used to solve for the instrument response by combining Eqs. (2) and (23) in Gardner et al. The fluorescence correction factor, planar fluoresce image, and probe concentration of the sample phantom, together with the known instrument response, are then used to solve for the unknown sample QY at each pixel. It is important to note that this methodology requires a stable absorption cross-section regardless of environmental conditions so that the probe concentration can be calculated despite any changes in QY. Both fluorophores used in this study fit these criteria for the absorption wavelengths used.

The QY of many fluorescent dyes is known to change under different environmental conditions. Previous studies have shown that the QY of rhodamine B is sensitive to solvent effects. ${ }^{7}$ In mixed methanol-water solvents, its QY varies from 0.52 to 0.30 while the methanol-water volume ratio changes from 100:0 to 0:100. ${ }^{7}$ SNARF-5 (Life Technologies, Carlsbad, California) exhibits a significant $\mathrm{pH}$-dependent emission shift between acidic and basic conditions and is commonly used as an intracellular $\mathrm{pH}$ indicator. ${ }^{4}$ Both dyes were used to validate QYI.

The absorption and emission spectra of both fluorophores were measured with a spectrophotometer (Cary 100 Bio UVVis, Varian, Palo Alto, California) and a fluorimeter (FluoroMax 3, Horiba, Kyoto, Japan), respectively. For rhodamine B, nine samples were made with varied methanol-water volume ratios (99:1, 90:10, 80:20, 70:30, 60:40, 50:50, 30:70, 10:90, and 0:100). For SNARF-5 measurements, nine samples with varied $\mathrm{pH}$ conditions were made $(\mathrm{pH}=6.07,6.39,6.82,7.11,7.79$, $8.13,8.36,8.88$, and 9.30). The fluorophore concentration was $3 \mu \mathrm{M}$ for all samples. For QYI experiments, measurements were conducted in liquid phantoms. The prepared liquid phantoms were poured into a $2.5 \times 2.5 \times 2 \mathrm{~cm}^{3}$ well embedded in a diffuse silicone phantom. In the rhodamine B experiments, the liquid phantoms had water/methanol mixtures as the solvent, nigrosin as absorber, and titanium dioxide as the scattering agent. For SNARF-5 experiments, phosphate buffered saline was used as solvent due to its low solubility in water. Nigrosin was used as the absorber and $1.1-\mu \mathrm{m}$ polystyrene microspheres as the scatterer, and $\mathrm{pH}$ was varied by adding either hydrochloric acid $(\mathrm{HCl})$ or sodium hydroxide $(\mathrm{NaOH})$. The background optical properties were adjusted by varying the amounts of absorbers and scatterers.

\section{Results}

\subsection{Quantum Yield Imaging for Rhodamine B}

Figure 2 shows the absorption spectrum of rhodamine B plotted from 450 to $650 \mathrm{~nm}$, and the emission spectrum from 561 to $660 \mathrm{~nm}$, with an excitation wavelength of $526 \mathrm{~nm}$. 526 and $561 \mathrm{~nm}$ correspond to the center wavelength of the illumination LED in both SFDI and fluorescence modes, and the cutoff wavelength of the long-pass filter in fluorescence mode, respectively. The solvent methanol-water ratio was varied to change the QY. Importantly, the absorption of rhodamine B was relatively stable while the emission changed dramatically with solvent.

To validate the QYI method, a series of 54 liquid phantoms were prepared, each with a different combination of solvent and optical properties. For simplicity, the concentration of rhodamine B was kept at $3 \mu \mathrm{M}$ for all liquid phantoms. This concentration was determined to be in the linear range of fluorescence emission, avoiding quenching and reabsorption effects. At each of the nine methanol-water mixture ratios (99:1, 90:10, 80:20, 70:30, 60:40, 50:50, 30:70, 10:90, and $0: 100)$, six liquid phantoms of varied optical properties were fabricated (Table 1). Note that the background optical properties of the liquid phantoms were measured without the presence of fluorescent dye, after which the dye was added to the liquid phantoms and then the fluorescence intensities were measured. The fluorophore concentration was calculated by comparing optical absorption at the excitation wavelength before and after adding the fluorophore, and with knowledge of rhodamine B's extinction coefficient at this wavelength. For each of the 54 liquid phantoms, the QY was determined by averaging within a region-of-interest corresponding to each liquid phantom and comparing these to literature values as shown in Fig. 3. Each colored dot represents a separate liquid phantom and different dot colors correspond to unique excitation wavelength background optical properties. The solid black line indicates known QYs from literature. ${ }^{7}$ Calculated QYs vary less than $10 \%$ from known values. Among the neighboring groups of the estimated QYs (grouped by solvent composition), the following pairs, 99:1/80:20, 80:20/60:40, 70:30/50:50, 50:50/30:70, 30:70/10:90, and 10:90/0:100, are statistically different, confirmed by student's $t$-test ( $p$-value $<0.05$ ). Calibration phantoms were made with a 50:50 methanol/water ratio.

The QYI method also allows spatial mapping of QY on pixelby-pixel basis. A series of nine liquid phantoms were made and
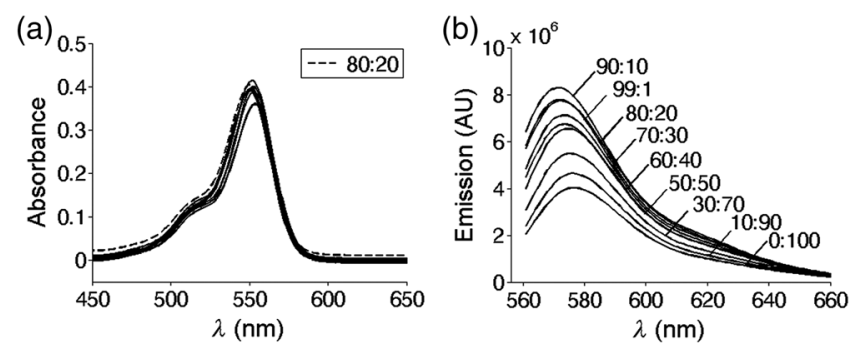

Fig. 2 Rhodamine B: (a) excitation and (b) emission spectra. 


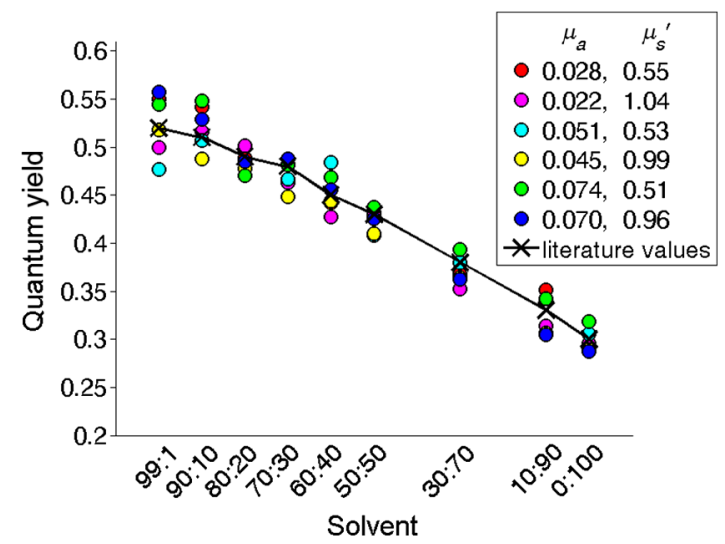

Fig. 3 Measured quantum yields versus literature values (rhodamine B). Lines were added between the standard values as a visual aid and do not represent interpolated data between measurements.

poured into nine wells embedded in a diffuse silicone phantom. These nine phantoms were imaged with SFDI and planar fluorescence, and pixel-level QY information was extracted as shown in Fig. 4. Figure 4(b) shows raw fluorescence, which is highest in the liquid phantoms with the lowest background absorption [(b), left column]. Figure 4(c) shows QY spatial mapping, demonstrating that correct QYs can be extracted regardless of background optical properties. The predicted and measured average QY in each well were within a $10 \%$ agreement on average. The image processing for spatial mapping was performed in MATLAB® (Mathworks, Natick, Massachusetts). The optical property maps of the background phantom were denoised by a close operation with a disk-shape template of 5-pixel radius, followed by median filtering with a 5-by-5 pixel neighborhood around each pixel. The computed QY maps were filtered by 10by-10 median filtering. For the rhodamine B experiments, the overall agreement for QY determination was 0.021 . This was calculated by determining the differences between the measured QYs and the literature values for each optical property combination, and then averaging over the entire experiment.

\subsection{Quantum Yield Imaging for SNARF-5}

The QY of SNARF-5 was measured under varying $\mathrm{pH}$ conditions to demonstrate the ability of QYI to perform as a noninvasive $\mathrm{pH}$ indicator. First, the absorbance and emission spectra of SNARF-5 were measured under different $\mathrm{pH}$ conditions in nondiffuse media using a spectrophotometer and fluorometer. Figure 5 shows the absorption spectrum of SNARF-5 collected from 400 to $700 \mathrm{~nm}$, and the emission spectrum from 561 to $850 \mathrm{~nm}$, with an excitation wavelength of $526 \mathrm{~nm}$. The QY of SNARF-5 was calculated by comparing these measurements to the spectra of a rhodamine B sample with 50:50 methanolwater solvent compositions. The rhodamine B sample has a known QY and was measured under the same spectrophotometer and fluorimeter settings. Results are listed in Table 2, which shows that the QY of SNARF-5 becomes larger with increasing $\mathrm{pH}$. Additionally, the QY plateaus at both high and low $\mathrm{pH}$ values.

QYI of SNARF-5 was tested in a $4 \times 4$ set of liquid phantoms, with four $\mathrm{pH}$ conditions ( $\mathrm{pH}=6.07,7.11,8.36$, and 9.30) and four different background optical property combinations. The $\mathrm{pH}$ was measured with a $\mathrm{pH}$ meter (Oyster-10, Extech Instruments, Nashua, New Hampshire). The optical properties of each of the four liquid phantoms are shown in Table 3. For simplicity, the concentration of SNARF-5 was kept at $3 \mu \mathrm{M}$ for all phantoms. QYs were extracted and compared to standard values, as shown in Fig. 6. Each colored dot in Fig. 6 represents a liquid phantom and dot color indicates a unique combination of background optical properties. Calculated QYs are within $20 \%$ of known values. This is inferior to the rhodamine B experiments (10\%), likely due to the fact that SNARF-5 QYs are generally much lower than those of rhodamine $\mathrm{B}$, leading to larger percent differences. Calibration phantoms had a $\mathrm{pH}$ of 7.79 .

To demonstrate spatial mapping of QY for SNARF-5, a series of four liquid phantoms were measured, and pixel-level QY information was extracted, as shown in Fig. 7. Figure 7(c) shows QYI spatial mapping. For the wells in (c), top row, the measured average QYs are 0.22 and 0.26 , with standard deviations (SD) of 0.025 and 0.024 , respectively. The expected QY for this row, based on spectrophotometer and fluorometer measurements, was 0.22 . The average measured QYs of the bottom row are $0.032(\mathrm{SD}=0.0031)$ and $0.038(\mathrm{SD}=0.0048)$, while the expected QY was 0.059. In general, SNARF-5 QYs were determined with agreement of 0.012 based on agreement calculations averaged over all phantoms. The optical property and QY maps were processed in the same manner as described for rhodamine $\mathrm{B}$ experiments.

To illustrate a dynamic, time-varying spatial mapping of QY, a $160-\mathrm{ml}$ liquid phantom was made in a $10.55 \times 6.82 \times$ $2.23 \mathrm{~cm}^{3}$ well embedded in a diffuse silicone phantom. SNARF-5 was added at a concentration of $3 \mu \mathrm{M}$, with phantom background optical properties of $\mu_{a}=0.016 \mathrm{~mm}^{-1}$ and $\mu_{s}^{\prime}=$ $0.533 \mathrm{~mm}^{-1}$ at $526 \mathrm{~nm}$. Sequential ml samples of $1 \mathrm{M} \mathrm{HCl}$ or $1 \mathrm{M} \mathrm{NaOH}$ were added from the upper left corner of the well (a)

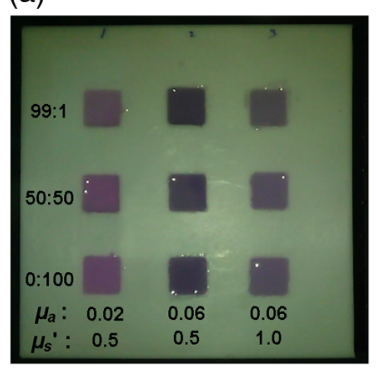

(b)

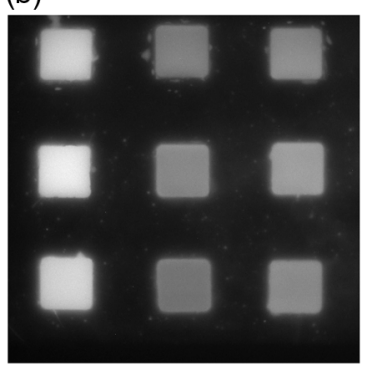

(c)

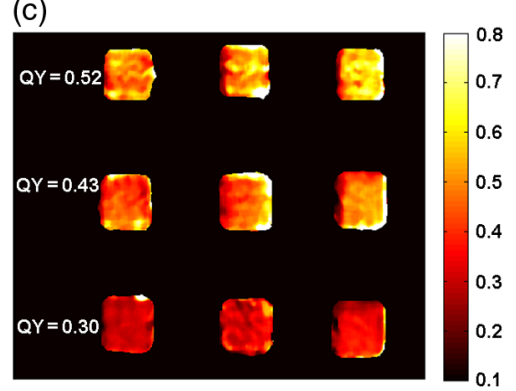

Fig. 4 Spatial mapping of quantum yield (rhodamine B): (a) white light image, (b) raw fluorescence, and (c) QY spatial mapping. 

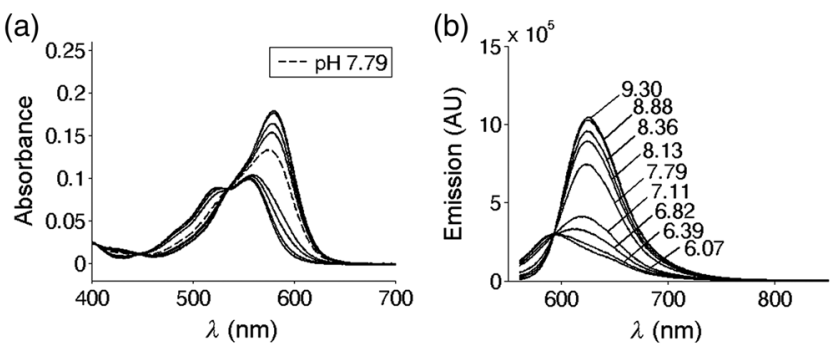

Fig. 5 SNARF-5: (a) excitation and (b) emission spectra.

Table 2 SNARF-5 quantum yields versus $\mathrm{pH}$.

\begin{tabular}{lc}
\hline $\mathrm{pH}$ & Quantum yield \\
\hline 6.07 & 0.059 \\
6.39 & 0.063 \\
6.82 & 0.074 \\
7.11 & 0.090 \\
7.79 & 0.151 \\
8.13 & 0.182 \\
8.36 & 0.195 \\
8.88 & 0.215 \\
9.30 & 0.221 \\
\hline
\end{tabular}

Table 3 Background optical properties of liquid phantoms (SNARF5).

\begin{tabular}{lll}
\hline & \multicolumn{2}{c}{ Excitation wavelength: $526 \mathrm{~nm}$} \\
\cline { 2 - 3 } Liquid phantoms & $\mu_{a}\left(\mathrm{~mm}^{-1}\right)$ & $\mu_{s}^{\prime}\left(\mathrm{mm}^{-1}\right)$ \\
\hline Phantom set 1 & 0.018 & 0.39 \\
Phantom set 2 & 0.015 & 0.81 \\
Phantom set 3 & 0.031 & 0.38 \\
Phantom set 4 & 0.029 & 0.79 \\
\hline
\end{tabular}

with a pipette. $\mathrm{HCl} / \mathrm{NaOH}$ diffused through the media, causing the local $\mathrm{pH}$ of the liquid phantom to change, and consequently the local QY. After the diffusion process, the liquid phantom was manually mixed and the $\mathrm{pH}$ was measured with a $\mathrm{pH}$ probe for validation. SFDI measurements were taken only once at baseline to determine the optical properties of the phantom, as well as the absorption caused by the introduction of the fluorescent dye. After that, the assumption was made that the optical properties were stable over time and only the planar fluorescent measurement was repeated, providing QY maps at 3-s intervals (each frame had an integration time of $2 \mathrm{~s}$ ).

In order to link sample $\mathrm{pH}$ values to QY measurements, a sigmoid function was used to fit to data from Table 2 . The fitted function is as follows:

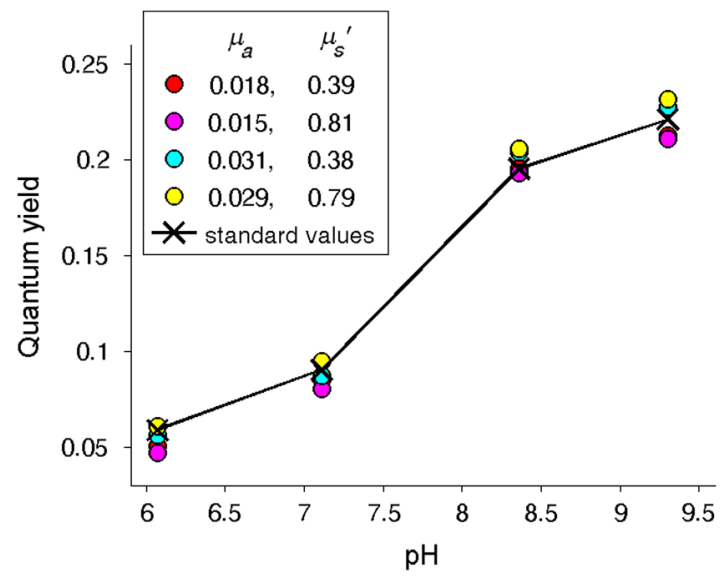

Fig. 6 Measured quantum yields versus standard values (SNARF-5). Lines were added between the standard values as a visual aid and do not represent interpolated data between measurements.

$$
\mathrm{QY}=0.0548+\frac{0.1692}{1+10^{1.0261(7.6727-\mathrm{pH})}}
$$

The $\mathrm{pH}$ of the sample was then extracted from QY values and a corresponding $\mathrm{pH}$ movie was created (Fig. 8 and Video 1). The $\mathrm{pH}$ was measured by the $\mathrm{pH}$ probe four times in total. Before adding any $\mathrm{HCl} / \mathrm{NaOH}$, the $\mathrm{pH}$ was measured as 7.54 , and the average $\mathrm{pH}$ from the extracted QYI $\mathrm{pH}$ map was 7.46 $(\mathrm{SD}=0.11$ ). After adding $0.64 \mathrm{ml} 1 \mathrm{M} \mathrm{HCl}$ and manual mixing, the $\mathrm{pH}$ was measured as 6.85 , and the average $\mathrm{pH}$ from the $\mathrm{pH}$ QYI map was $6.74(\mathrm{SD}=0.17)$. Then after adding another $0.64 \mathrm{ml} 1 \mathrm{M} \mathrm{HCl}$ and the manual mixing, the $\mathrm{pH}$ was measured as 6.17, and the average $\mathrm{pH}$ from the $\mathrm{pH}$ map was 4.58 ( $\mathrm{SD}=$ $0.55)$. In the end after adding $1.28 \mathrm{ml} 1 \mathrm{M} \mathrm{NaOH}$ and manual mixing, the $\mathrm{pH}$ was measured as 7.54, and the average $\mathrm{pH}$ from the $\mathrm{pH}$ map was $7.41(\mathrm{SD}=0.16)$. This dynamic process is shown in Video 1; Fig. 8 shows two frames from the movie.

\section{Discussion}

We have demonstrated a new imaging method, called QYI, which uses SFDI and planar fluorescence to extract QY values from a fluorescent dye within an optically diffusive background. QYI extends prior quantitative fluorescence methods which assume a constant and known QY for a given fluorophores of interest. ${ }^{13-15,18}$ By separately measuring fluorophore concentration in the relatively stable absorption band, we are able to determine the QY of the fluorophore by correcting the raw planar fluorescence signal in the emission band, where fluorescence intensity changes greatly with environmental conditions. QYI was demonstrated using two fluorophores, rhodamine B and SNARF-5, which are sensitive to solvent and $\mathrm{pH}$ conditions, respectively. The QY of these fluorophores was measured and validated in optical phantoms with different background optical properties. Additionally, pixel-level mapping of optical properties with SFDI allowed for spatial mapping of QY for both of the fluorophores. Finally, Video 1 was made showing dynamic changes of both QY and environmental $\mathrm{pH}$ values using the SNARF-5 dye.

The QY-pH relationship suggests in vivo spatial mapping of local tissue $\mathrm{pH}$ as one of the potential future applications of this method. Due to absorption and scattering, measuring $\mathrm{pH}$ and other parameters is currently difficult in thick tissue. For example, $\mathrm{pH}$ is currently measured in tissue with either invasive 
(a)

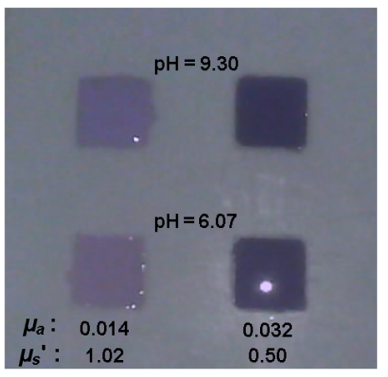

(b)

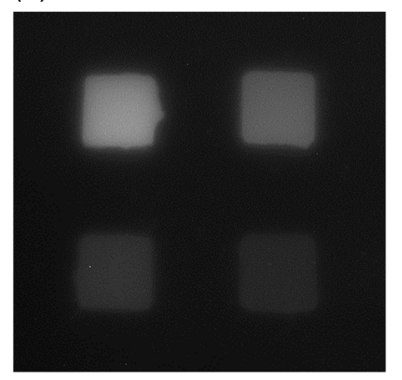

(c)

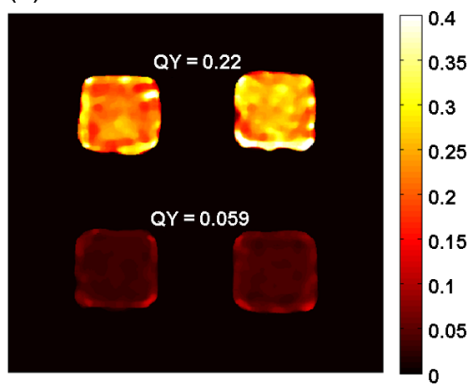

Fig. 7 Spatial mapping of quantum yield (SNARF-5): (a) white light image, (b) raw fluorescence, and (c) QY spatial mapping.

probes or ratiometric $\mathrm{pH}$ dyes. ${ }^{22,23}$ Invasive probes have limited applicability to longitudinal studies, and ratiometric dyes may be unreliable in thick tissue due to the wavelength dependence of optical properties. In addition, invasive probes are applicable for measuring $\mathrm{pH}$ at only a single or a few locations in tissue. There has been some work done in using fluorescence lifetime measurements to determine $\mathrm{pH}$ conditions in tissue, but this requires relatively complex and expensive pulsed laser systems and time-correlated single photon counting techniques. ${ }^{24-27}$ Moreover, fluorophores which are sensitive to temperature, oxygen content, or protein binding can also be used with QYI, potentially allowing a variety of microenvironmental conditions to be determined noninvasively.

Several assumptions were made in this study in order to simplify the processing required to generate QYI maps, and there are limitations to the use of this methodology for certain applications. For example, the Gardner model assumes homogeneity in depth, which may limit the method's use for some in vivo applications. Additionally, QYI requires the use of a dye with a stable absorption band, which was the case for both rhodamine B and SNARF-5 at the wavelengths used here, but does not apply for all molecular imaging agents. Additionally, SFDI measurements were required before and after adding the fluorophore to the diffuse background in order to determine the probe concentration prior to calculating the QY, limiting the use of QYI for some real-time applications. The agreement between expected and measured QY was within $10 \%$ for rhodamine B experiments and within $20 \%$ for most SNARF-5 experiments, validating the calibration procedures used for this study, although care should be taken in future investigations in regards to more complex sample geometry, collection geometry, and wider ranges of fluorophore concentrations. Despite these requirements and limitations, QYI imaging should still be useful for in vivo preclinical imaging, and the methodology is amenable to the use of more complex quantitative fluorescence models that would allow for tomographic reconstructions with depth resolved information. Additionally, these same methods are applicable to quantitative spectroscopic modalities such as (a)

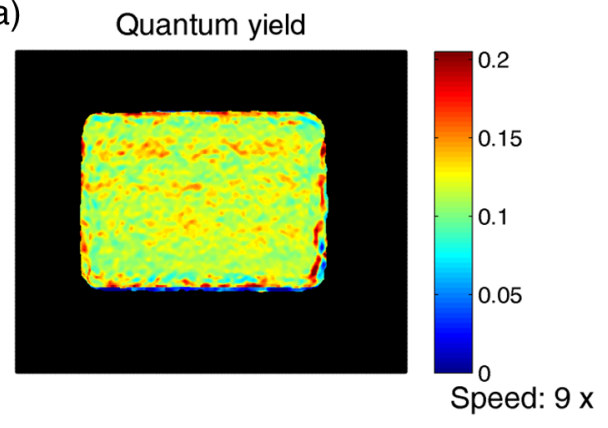

(b)
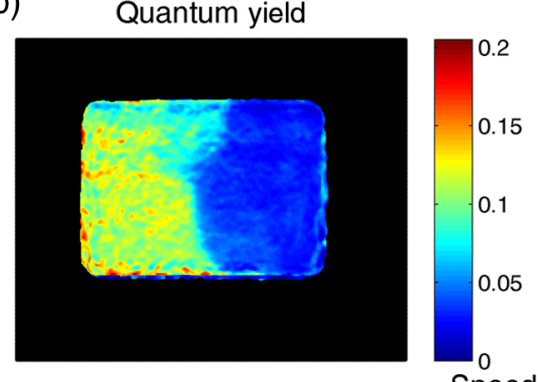

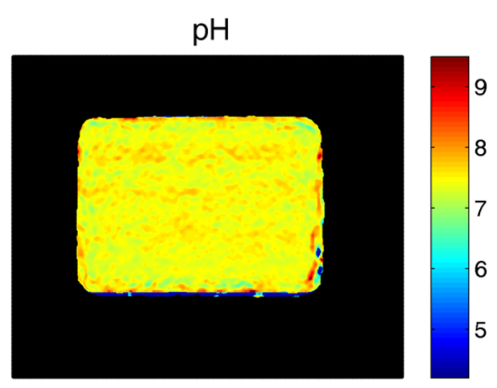

$\mathrm{pH}$

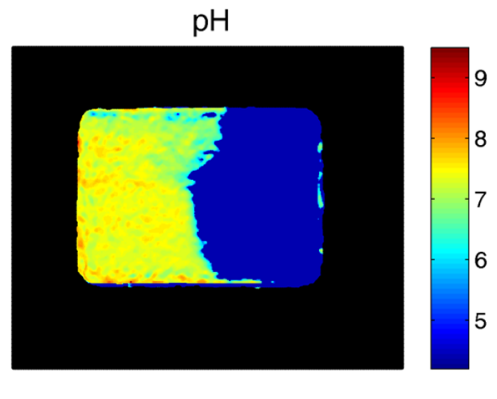

Fig. 8 (a) and (b) Dynamic spatial mapping of quantum yield and pH. Text notes signify each step above during video playback. (Video 1, MPEG, 3.19 MB) [URL: http://dx.doi.org/10.1117/1.JBO.20.8 $.086013 .1]$. 
frequency and time-domain diffuse optical systems if combined with fluorescent measurements, providing deeper tissue depth penetration and possible in vivo human applications.

In summary, QYI is able to extract fluorophore QY regardless of background optical properties, highlighting the possibility of using QYI with environmentally sensitive fluorophores to create novel biomarkers to probe tissue microenvironment in vivo.

\section{Acknowledgments}

The authors wish to thank Irving Bigio, Amaan Mazhar, David Cuccia, and Bruce Tromberg for their helpful discussions. The authors gratefully acknowledge funding from the American Cancer Society (Grant RSG-14-014-01-CCE).

\section{References}

1. R. Weissleder and V. Ntziachristos, "Shedding light onto live molecular targets," Nat. Med. 9(1), 123-128 (2003).

2. K. Rurack and M. Spieles, "Fluorescence quantum yields of a series of red and near-infrared dyes emitting at 600-1000 nm," Anal. Chem. 83, 1232-1242 (2011).

3. J. Laverdant et al., "Experimental determination of the fluorescence quantum yield of semiconductor nanocrystals," Materials (Basel) 4(7), 1182-1193 (2011).

4. J. Han and K. Burgess, "Fluorescent indicators for intracellular $\mathrm{pH}$," Chem. Rev. 110, 2709-2728 (2010).

5. D. Magde, G. E. Rojas, and P. G. Seybold, "Solvent dependence of the fluorescence lifetimes of xanthene dyes," Photochem. Photobiol. 70(5), 737-744 (1999).

6. D. Magde, R. Wong, and P. G. Seybold, "Fluorescence quantum yields and their relation to lifetimes of rhodamine $6 \mathrm{G}$ and fluorescein in nine solvents: improved absolute standards for quantum yields," Photochem. Photobiol. 75(4), 327-334 (2002).

7. T. L. Chang and H. C. Cheung, "Solvent effects on the photoisomerization rates of the zwitterionic and the cationic forms of rhodamine B in protic solvents," J. Phys. Chem. 96, 4874-4878 (1992).

8. W. R. Ware and B. A. Baldwin, "Effect of temperature on fluorescence quantum yields in solution," J. Chem. Phys. 43(4), 1194 (1965).

9. A. M. Brouwer, "Standards for photoluminescence quantum yield measurements in solution (IUPAC technical report)," Pure Appl. Chem. 83(12), 2213-2228 (2011).

10. G. A. Crosby and J. N. Demas, "Measurement of photoluminescence quantum yields. Review," J. Phys. Chem. 75, 991-1024 (1971).

11. T. Karstens and K. Kobs, "Rhodamine B and rhodamine 101 as reference substances for fluorescence quantum yield measurements," J. Phys. Chem. 84(14), 1871-1872 (1980).

12. J. Olmsted, "Calorimetric determinations of absolute fluorescence quantum yields," J. Phys. Chem. 83(20), 2581-2584 (1979).

13. J. Wu, M. S. Feld, and R. P. Rava, "Analytical model for extracting intrinsic fluorescence in turbid media," Appl. Opt. 32(19), 3585-3595 (1993).

14. C. M. Gardner, S. L. Jacques, and A. J. Welch, "Fluorescence spectroscopy of tissue: recovery of intrinsic fluorescence from measured fluorescence," Appl. Opt. 35, 1780-1792 (1996).
15. C. M. Gardner, S. L. Jacques, and A. J. Welch, "Light transport in tissue: accurate expressions for one-dimensional fluence rate and escape function based upon Monte Carlo simulation," Lasers Surg. Med. 18, 129138 (1996).

16. D. J. Cuccia et al., "Quantitation and mapping of tissue optical properties using modulated imaging," J. Biomed. Opt. 14, 024012 (2009).

17. M. Martinelli et al., "Analysis of single Monte Carlo methods for prediction of reflectance from turbid media," Opt. Express 19(20), 19627 (2011).

18. R. B. Saager et al., "Quantitative fluorescence imaging of protoporphyrin IX through determination of tissue optical properties in the spatial frequency domain," J. Biomed. Opt. 16(12), 126013 (2011).

19. S. D. Konecky et al., "Quantitative optical tomography of sub-surface heterogeneities using spatially modulated structured light," Opt. Express 17(17), 14780-14790 (2009).

20. S. D. Konecky et al., "Spatial frequency domain tomography of protoporphyrin IX fluorescence in preclinical glioma models," J. Biomed. Opt. 17(5), 056008 (2012).

21. S. Gioux et al., "Three-dimensional surface profile intensity correction for spatially modulated imaging," J. Biomed. Opt. 14(3), 034045 (2009).

22. R. Pal and D. Parker, "A ratiometric optical imaging probe for intracellular $\mathrm{pH}$ based on modulation of europium emission," Org. Biomol. Chem. 6(6), 1020-1033 (2008).

23. S. Mordon et al., "Characterization of tumorous and normal tissue using a pH-sensitive fluorescence indicator (5, 6-carboxyfluorescein) in vivo," J. Photochem. Photobiol. B Biol. 13, 307-314 (1992).

24. I. Gannot et al., "Functional optical detection based on $\mathrm{pH}$ dependent fluorescence lifetime," Lasers Surg. Med. 35(5), 342-348 (2004).

25. G. R Martin and R. K. Jain, "Fluorescence ratio imaging measurement of $\mathrm{pH}$ gradients: calibration and application in normal and tumor tissues," Microvasc. Res. 46(2), 216-230 (1993).

26. J. E. Mathejczyk et al., "High-sensitivity detection of breast tumors in vivo by use of a $\mathrm{pH}$-sensitive near-infrared fluorescence probe," $J$. Biomed. Opt. 17, 076028 (2012).

27. D. Y. Paithankar et al., "Imaging of fluorescent yield and lifetime from multiply scattered light reemitted from random media," Appl. Opt. 36, 2260-2272 (1997).

Yanyu Zhao received his BS degree in optoelectronic information engineering from Beihang University (previously known as Beijing University of Aeronautics and Astronautics) in Beijing, China. Currently, he is a PhD candidate in the Department of Biomedical Engineering at Boston University. His research interests include quantitative diffuse optical imaging and data analysis for biomedical applications.

Darren Roblyer is an assistant professor in the Department of Biomedical Engineering at Boston University. After receiving his BS degree in biomedical engineering from Johns Hopkins University in 2004, Darren received his $\mathrm{PhD}$ in 2009 from the Bioengineering Department at Rice University. Prior to starting his faculty position, Darren was a Department of Defense postdoctoral fellow at the Beckman Laser Institute at the University of California, Irvine. He is a member of SPIE. 